\title{
Comparison of neck muscle electromyography activity in response to external force between static and dynamic loading
}

\begin{abstract}
Understanding the behavior of neck muscles is essential to accurately simulate the human head-neck segment movement especially for low-speed motor vehicle crash situation. Some head-neck mathematical models were designed using neck muscle activation behavior in isometric contraction (static loading) as the properties of neck muscle activation. However, neck muscle activation pattern and strength capability may vary between static and dynamic loading. This study aimed to determine the differences between neck muscle activation level under static and dynamic loading. A neck strength test involving 22 human volunteers was conducted with two different tasks in extension and flexion direction with three different loads. The neck muscle activation level is determined through measuring the electromyography (EMG) responses of selected flexor and extensor muscles using surface bilateral electrode and recorded. The findings showed that neck muscle activation level was significantly greater in dynamic loading than static loading $(\mathrm{p}<0.000)$. These implied that more efforts from neck muscles were required to resist against dynamic loading than static loading. Nonetheless, the differences in EMG activities between these two loading conditions progressively decreased when more loads were applied. This study has established an empirical model to describe the relationship between neck muscle activation level and force output for both loading condition in flexion and extension.
\end{abstract}

Keyword: Neck muscle; Muscle activation level; Neck muscle force 